九州大学学術情報リポジトリ

Kyushu University Institutional Repository

\title{
The Influence of Magnetic Field on Muography Images: A Simulation Study
}

Basiri, Hamid

Interdisciplinary Graduate School of Engineering Sciences, Kyushu University

Kin, Tadahiro

Interdisciplinary Graduate School of Engineering Sciences, Kyushu University

Okamoto, Naoya

Interdisciplinary Graduate School of Engineering Sciences, Kyushu University

https://doi.org/10.5109/4738558

出版情報: Proceedings of International Exchange and Innovation Conference on Engineering \& Sciences (IEICES). 7, pp. 45-50，2021-10-21. 九州大学大学院総合理工学府 バージョン：

権利関係: 


\title{
The Influence of Magnetic Field on Muography Images: A Simulation Study
}

\author{
Hamid Basiri ${ }^{*}$, Tadahiro Kin ${ }^{1}$, Naoya Okamoto ${ }^{1}$ \\ ${ }^{1}$ Interdisciplinary Graduate School of Engineering Science, Kyushu University, 6-1 Kasuga-koen, Kasuga-shi, Fukuoka, \\ 816-8580, Japan. \\ "Corresponding author email: basiri.hamid.218@s.kyushu-u.ac.jp
}

\begin{abstract}
Muography or muon radiography is a non-invasive, emerging imaging technique based on the analysis of the absorption or scattering of atmospheric muons. This technique has been used in various applications, such as imaging an unreachable object, such as a damaged nuclear reactor or magma in a volcano and searching for hidden cavities in pyramids. The applications of muography are becoming broader, and new techniques are being developed. In this work, the authors investigated the influence of the magnetic field on a muography image and the trajectories of muons through simulations using the PHITS Monte Carlo code. The results showed that the magnetic field can distort the muography image by changing the trajectories of the muons due to the influence of the Lorentz force, and this distortion should be taken into account in any muography application that involves the magnetic field.
\end{abstract}

Keywords: Cosmic-ray muon, Muography, Muon radiography, Magnetic field

\section{INTRODUCTION}

Muons are elementary particles similar to electrons with a mass about 200 times greater. These secondary cosmic ray particles are generated as a result of the interaction of primary cosmic rays with the upper atmosphere [1]. Muons are the most numerous energetic particles to reach the sea level, and their flux has been reported to be about 1 muon per square centimeter per minute. The average energy of muons arriving at sea level is about $4 \mathrm{GeV}$ with a lifetime of $2.2 \mu \mathrm{s}$ [2]. This high energy allows them to penetrate deeply into any material and they can travel several hundred meters through the Earth as a relativistic particle.

Cosmic-ray muons represent a free and available source at any point on Earth. Their special properties, such as deep penetrating power, encourage researchers to develop a non-destructive technique to study various targets. This method is known as muon tomography technique or muography and has been developed for different type of applications [3].

In some applications, cosmic muons have been treated in the same way as X-rays in ordinary radiography. By analyzing the absorbed fraction of muons as they traverse a volume, information about the volume can be obtained. The first time the depth of the rock layer above an underground tunnel in Australia was measured in the 1950s Eric George by studying the absorption of muons [4]. Then, in the 1970s, Alvarez proposed this method to study the pyramid for hollow vaults [5]. More recently, muography has been successfully used in a variety of fields, including inspection of the internal structures of volcanoes [6-10], detection of heavy metals in shipping containers, inspection of nuclear smuggling [11-13], mining [14], geological and archeological studies $[15,16]$, investigation of infrastructure degradation [17], and monitoring of reactors and nuclear waste [18-21].

In all the above applications, the image of muography can be distorted when muons cross a magnetic field region, and the effect of a magnetic field on the bending angle of muons has not been considered in any previous study. In this work, we will use simulations to show that this effect is important and can affect the muography image.

\section{MUOGRAPHY IMAGING TECHNIQUES}

Muons, being charged particles, interact with the atoms of matter (mainly by ionization) while passing through it. Their energy loss or change of direction (scattering angle) is proportional to the thickness of matter they traverse. Two muography techniques have been developed by scientists based on the analysis of the scattering of muons or their absorption in matter [22].

\subsection{Muon Scattering Method}

This muography technique is based on multiple Coulomb scattering of muons as they traverse the volume of the region of interest. Muons are deflected and slowed down when they interact with a material with a high atomic number. By using two sets of position sensitive detectors before and after the volume of interest, the deflection angles can be measured, and then high-Z objects can be distinguished from other materials [23]. This method is fast and hazard-free. For example, $20 \mathrm{~kg}$ of uranium hidden in more than 10 tons of iron can be detected in less than a minute by analyzing the scattering angles of muons. The scattering angle distribution can be described by Moliere theory [24] and is approximately given by a Gaussian with zero mean and standard deviation:

$\theta_{M S}=\frac{13.6 \mathrm{MeV}}{\beta c p} Q \sqrt{\frac{x}{X_{0}}}\left(1+0.038 \ln \left(\frac{x}{X_{0}}\right)\right)$

where $\beta, p, x, Q, X_{0}$ represent the relativistic factor, the muon momentum in $M e V / c$, the material thickness, the absolute electric charge of the muon and the radiation length, respectively. The radiation length is empirically given by:

$X_{0} \approx \frac{716.4 \mathrm{~g} / \mathrm{cm}^{2}}{\rho} \frac{A}{Z(Z+1) \log \left(\frac{287}{\sqrt{(Z)}}\right)}$

where $\mathrm{Z}$ and $A$ represent the atomic and mass numbers respectively.

\subsection{Muon Absorption Method}

Muons are subatomic particles that can penetrate deeply 
into any material because of their high energy. Muons are sensitive to changes in the density of the materials they penetrate. Thus, their absorption rate depends on this density. By measuring the muon flux rate in different directions, the density changes of the materials between the muon source and the detector can be provided.

The intensity of the cosmic muons can be represented by the measured count rate. If we consider $R_{0}$ as the count rate of muons in a detector before the region of interest and $\mathrm{R}$ as the count rate after passing through the absorbing medium, we obtain:

$$
R=R_{0} e^{-\mu x}
$$

if the count rate and the thickness of the absorber are measured, the absorption coefficient can be determined from the slope obtained after fitting the linear equation,

$\ln (R)=\ln \left(R_{0}\right)-\mu x$

This equation can be used to experimentally determine the absorption coefficient. The fitting adapted is the least square technique [25].

\section{Muons in Magnetic field}

Muons, like other charged particles, feel a Lorentz force when they enter a magnetic field region:

$\vec{F}=q \vec{v} \times \vec{B}$

where $\vec{F}, q, \vec{v}$, represent the Lorentz force, the charge of particle, the velocity of the particle, respectively, and $\vec{B}$ is the magnetic flux intensity.

The change in muon tracks depends on the muon energy (velocity term) and the magnitude of the magnetic field flux. Fig. 1 shows a positive muon traversing a magnetic field region. The direction of the muon can be determined by the right-hand rule.

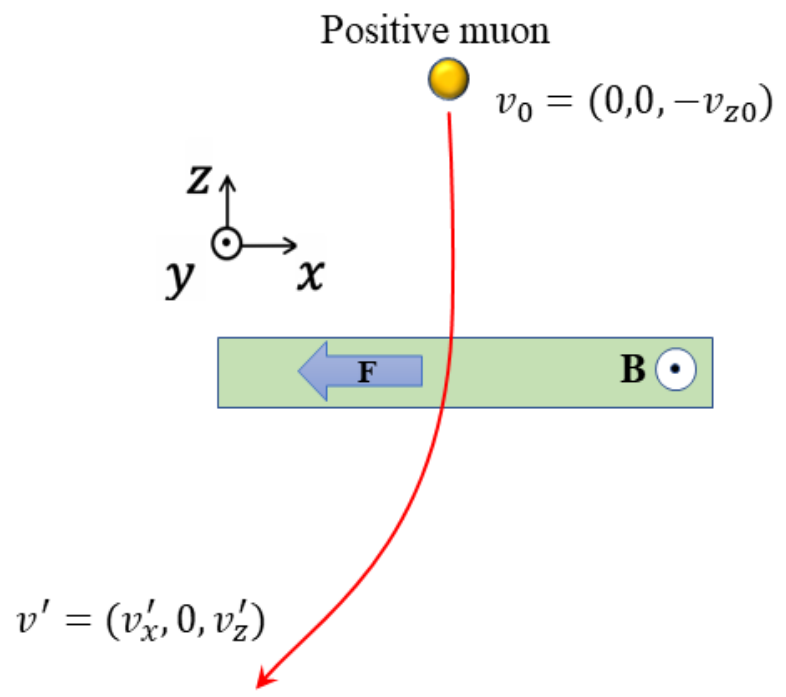

Fig. 1: Muon trajectory in magnetic field

\section{Monte Carlo Simulations}

To investigate the effect of the magnetic field on the trajectories of the muons, simulations were performed using the PHITS (Particle and Heavy-Ion Transport code System) Monte Carlo code [26] in three different steps.

In the first step, a magnetic region of vacuum with a size of $300 \mathrm{~cm} \times 100 \mathrm{~cm} \times 100 \mathrm{~cm}$ and a magnetic flux density of $500 \mathrm{mT}$ was defined. A mono energy surface source of positive and negative muons was generated, and the track of the muons was investigated. Fig. 2 and 3 represent the changes in the muon tracks due to the magnetic field for negative and positive muons, respectively.

Then, mono-energy point source muons were defined and their changes due to traversing a $1 \mathrm{~m}$ thick magnetic region were studied. In this step, simulations were performed for $3 \mathrm{GeV}, 1 \mathrm{GeV}$, and $500 \mathrm{MeV}$ muons in $100 \mathrm{mT}, 500 \mathrm{mT}$, and $1 \mathrm{~T}$, respectively, to compare the effect of magnetic field strength and muon energy on muon tracks.

In the next step, the muons traversed a region of dimension $300 \mathrm{~cm} \times 100 \mathrm{~cm} \times 50 \mathrm{~cm}$ of iron in two states; in the first state, this area is not set to be a magnetic material and in the second state, the area defined as magnetic material with a magnitude of flux density of $500 \mathrm{mT}$.

In the final simulation, the muography image of 10 iron plates was obtained in 2 states: by normal muography and then by implementing a quadrupole magnetic region in front of the detection plane.

\section{Results}

Fig.s 2 and 3 shows the results for a surface beam with negative and positive muons respectively. Fig. 4 represents the muons' tracks for both negative and positive muons. As we expect from theory, the change in muons' tracks as a result of Lorentz force can be observed.

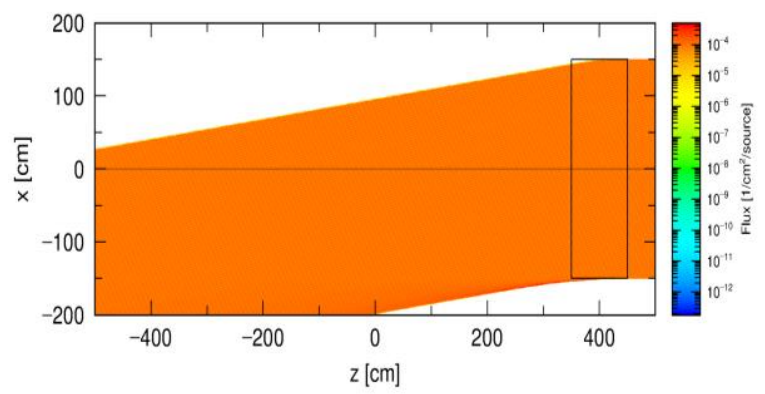

Fig. 2: Effect of $500 \mathrm{mT}$ magnetic field on $1 \mathrm{GeV}$ negative muons' tracks 


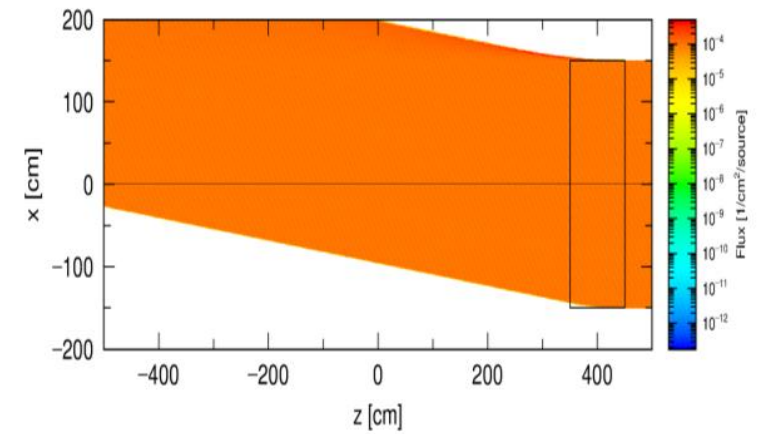

Fig. 3: Effect of $500 \mathrm{mT}$ magnetic field on $1 \mathrm{GeV}$ positive muons' tracks

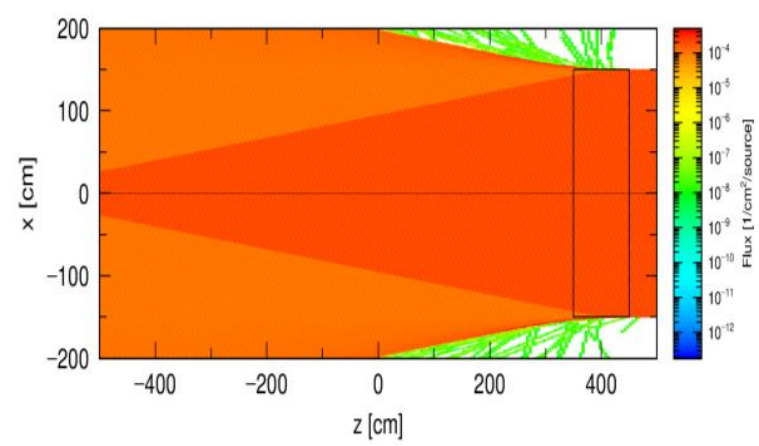

Fig. 4: Effect of a $500 \mathrm{mT}$ magnetic field on the tracks of $1 \mathrm{GeV}$ positive and negative muons.
Fig. 5 shows the results of the simulations for a monoenergetic point source with different energies when passing through different magnetic fields. The change in the tracks of the muons is well seen. As expected from the theory, a lower energy or a stronger magnetic field led to a larger bending angle for the muons.

Until the previous step, the material of the magnetic region was set to vacuum. For the next step, we change the thickness of the magnetic field to $50 \mathrm{~cm}$ and the material to iron. Fig. 6 shows a comparison for two conditions, considering the target as normal iron and as a magnet with a magnetic flux density of $500 \mathrm{mT}$.

Finally, Figs. 7 and 8 represent the simulation geometry and the muography image of 10 iron plates in normal situation and while crossing a quadrupole magnet region. The distortion can be seen in the second scenario.
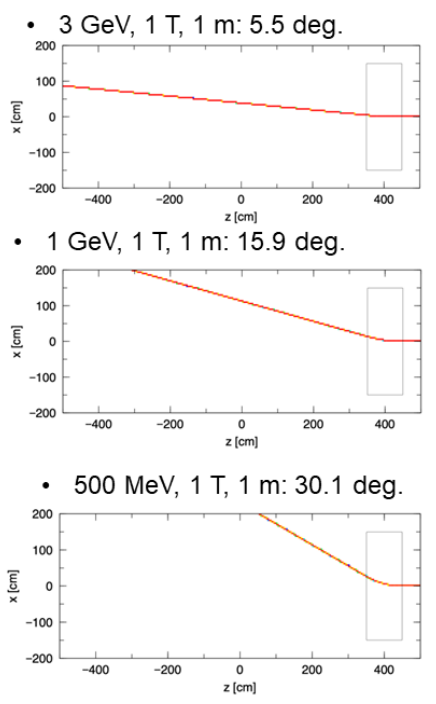

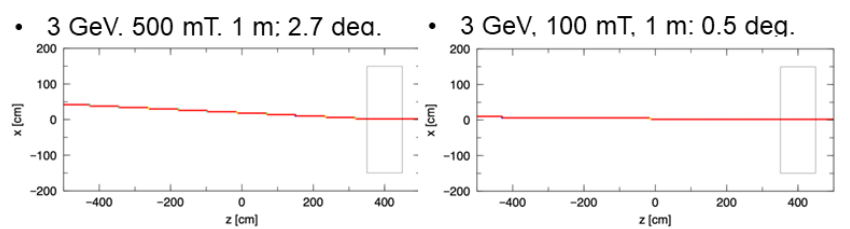

- $1 \mathrm{GeV}, 500 \mathrm{mT}, 1 \mathrm{~m}: 7.9 \mathrm{deg}$.
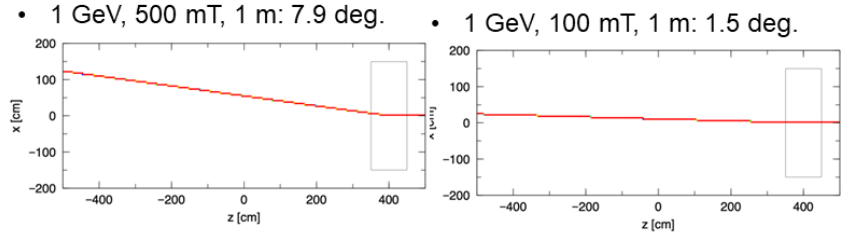

- $500 \mathrm{MeV}, 500$ mT, 1 m: 14.5 deg.
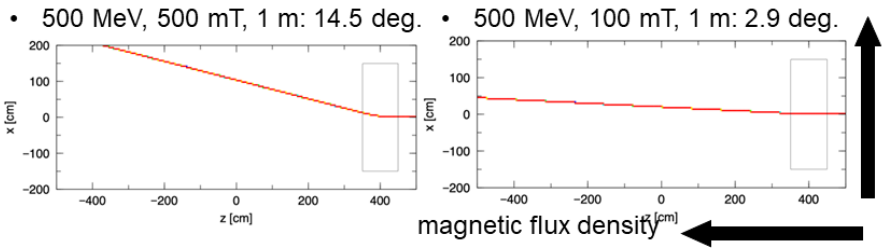

Fig. 5: Comparison of the effect of the magnetic field and the muon energy on the tracks of the muons. 


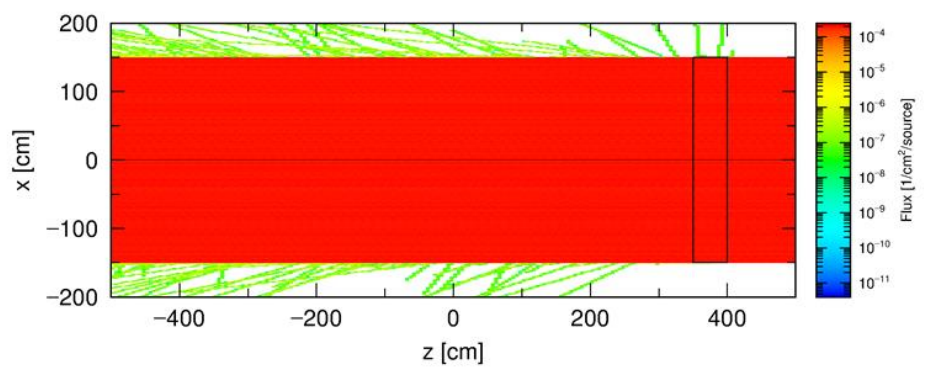

(a)

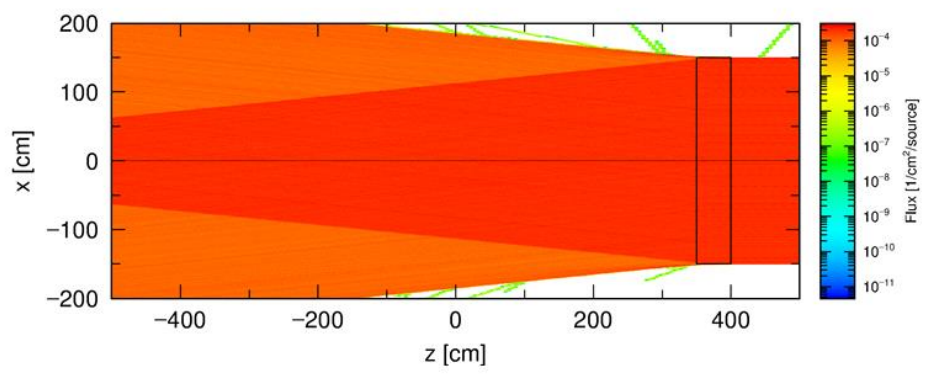

(b)

Fig. 6: Comparison of the muon tracks when crossing (a) an iron target and (b) a magnetic field of $500 \mathrm{mT}$ in a region of iron.

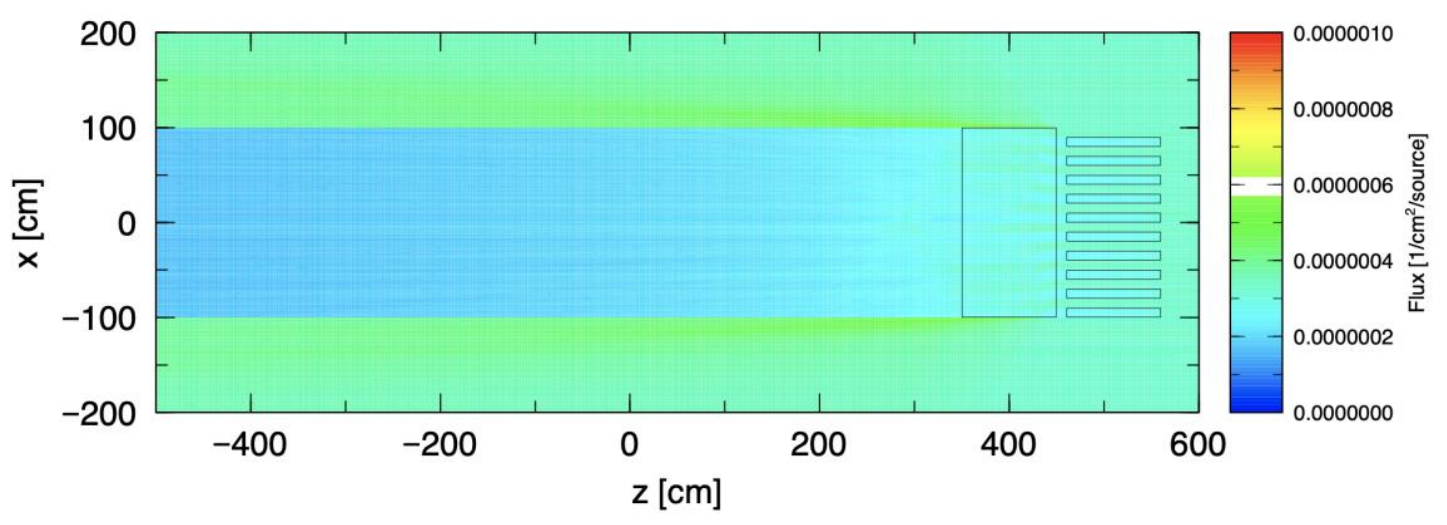

Fig. 7: Simulation used for muography image of 10 iron plates.

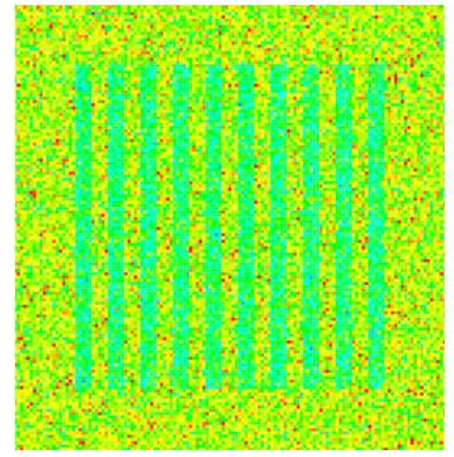

(a)

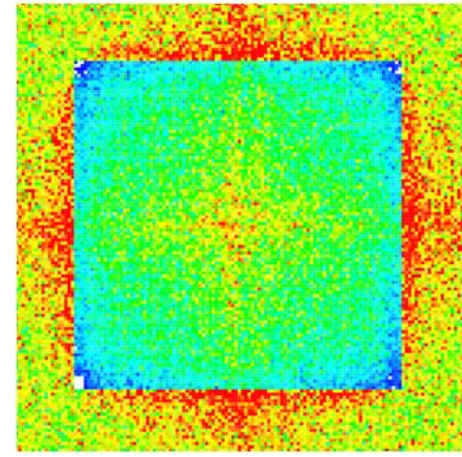

(b)

Fig. 8: Muography image of 10 iron plates for (a) normal muography and (b) in the presence of a magnetic field.

\section{Discussion}

Simulations showed that the magnetic field can change the tracks of the muons and the bending angle of the muons depends on the muon energy and the flux density of the magnetic field. A lower muon energy and a stronger magnetic field led to more changes in the muon trajectories, and they were bent more. The flux of muons in the detection plane region can also change, as we saw in the simulation results. 
In summary, changes in the muon trajectories due to Lorentz force caused by magnetic region, can act as a noise on muography image. In any muography application, this important factor of whether the muons have crossed a magnetic region or not should be taken into account. Also, in some muography applications, such as materials inspection by comparing the average of the scattering angles of muons, the change in the muon scattering angle can lead to an incorrect result, and correction is necessary to eliminate this effect.

\section{REFERENCES}

[1] Nagamine, K., Introductory Muon Science. 2003: Cambridge University Press.

[2] Cox, L., et al. Detector requirements for a cosmic ray muon scattering tomography system. in Nuclear Science Symposium Conference Record, 2008. NSS'08. IEEE. 2008. IEEE.

[3] L. Bonechi, R. D'Alessandro, and A. Giammanco, "Atmospheric muons as an imaging tool," Rev. Phys., vol. 5, no. June 2019, p. 100038, 2020, doi: 10.1016/j.revip.2020.1000

[4] George E. 1955. Cosmic rays measure overburden of tunnel. Commonwealth Engineer 1955, 455-457.

[5] L. W. Alvarez et al., "Search for hidden chambers in the pyramids," Science (80-. )., vol. 167, no. 3919, pp. 832-839, 1970, doi: 10.1126/science.167.3919.832.

[6] R. D'Alessandro et al., "Volcanoes in Italy and the role of muon radiography," Philos. Trans. R. Soc. A Math. Phys. Eng. Sci., vol. 377, no. 2137, p. 20180050, Jan. 2019, doi: 10.1098/rsta.2018.0050.

[7] A. Anastasio et al., "The MU-RAY experiment. An application of SiPM technology to the understanding of volcanic phenomena," Nucl. Instruments Methods Phys. Res. Sect. A Accel. Spectrometers, Detect. Assoc. Equip., vol. 718, pp. 134-137, 2013, doi: 10.1016/j.nima.2012.08.065.

[8] L. Oláh, H. K. M. Tanaka, G. Hamar, and D. Varga, "Investigation of the limits of high-definition muography for observation of Mt Sakurajima," Philos. Trans. R. Soc. A Math. Phys. Eng. Sci., vol. 377, no. 2137, 2019, doi: 10.1098/rsta.2018.0135.

[9] H. K. M. Tanaka, "Japanese volcanoes visualized with muography," Philos. Trans. R. Soc. A Math. Phys. Eng. Sci., vol. 377, no. 2137, 2019, doi: 10.1098/rsta.2018.0142.

[10] D. Carbone, D. Gibert, J. Marteau, M. Diament, L. Zuccarello, and E. Galichet, "An experiment of muon radiography at Mt Etna (Italy),” Geophys. J. Int., vol. 196, no. 2, pp. 633-643, Jan. 2013, doi: 10.1093/gji/ggt403.

[11] MU-BLAST. 2014. Study of the capability of muon tomography to map the material composition inside a blast furnace. EU publications Research found for coal and steel RFSR-CT-2014-00027.
[12] Xianfeng H. et al. 2018. Exploring the capability of muon scattering tomography for imaging the components in the blast furnace. ISIJ Int. 58, 35-42. (10.2355/isijinternational.ISIJINT-2017-384)

[13] Aström E. et al. 2016. Precision measurements of linear scattering density using muon tomography. J. Instrum. 11, P07010 (10.1088/1748-0221/11/07/p07010

[14] Zhang, Z.X., Enqvist, T., Holma, M. and Kuusiniemi, P., 2020. Muography and its potential applications to mining and rock engineering. Rock Mechanics and Rock Engineering, 53(11), pp.4893-4907.

[15] Gómez H, Carloganu C, Gibert D, Jacquemier J, Karyotakis Y, Marteau J, Niess V, Katsanevas S, Tonazzo A. 2016. Studies on muon tomography for archaeological internal structures scanning. J. Phys: Conf. Ser. 718, 052016

[16] Dedenko LG. et al. 2014. Prospects of the study of geological structures by muon radiography based on emulsion track detectors. Bull. Lebedev Phys. Inst. 41, 235-241.

[17] K. Chaiwongkhot et al., "Development of a Portable Muography Detector for Infrastructure Degradation Investigation," IEEE Trans. Nucl. Sci., vol. 65, no. 8, pp. 2316-2324, Aug. 2018.

[18] H. Miyadera et al., "Imaging Fukushima Daiichi reactors with muons," AIP Adv., vol. 3, no. 5, p. 052133, May 2013, doi: 10.1063/1.4808210.

[19] J. Perry et al., "Imaging a nuclear reactor using cosmic ray muons," J. Appl. Phys., vol. 113, no. 18, 2013 , doi: 10.1063/1.4804660.

[20] K. Morishima et al., "First demonstration of cosmic ray muon radiography of reactor cores with nuclear emulsions based on an automated high-speed scanning technology," Proc. 26th Work. Radiat. Detect. Their Uses, pp. 27-36, 2012.

[21] D. Poulson, J. Bacon, M. Durham, E. Guardincerri, C. L. Morris, and H. R. Trellue, "Application of muon tomography to fuel cask monitoring," Philos. Trans. R. Soc. A Math. Phys. Eng. Sci., vol. 377, no. 2137, 2019, doi: 10.1098/rsta.2018.0052.

[22] Procureur, S., 2018. Muon imaging: Principles, technologies and applications. Nuclear Instruments and Methods in Physics Research Section A: Accelerators, Spectrometers, Detectors and Associated Equipment, 878, pp.169-179.

[23] K. Borozdin et al., Cosmic-ray muon tomography and its application to the detection of high- $Z$ materials. Nature 422, 277 (2003).

[24] H.A. Bethe. Molière's Theory of Multiple Scattering, Physical Review 89 (1953) 1256 
[25] Altameemi, R.N., Abdul Hamid, N.S., Wan Mohd Kamil, W.M.A. and Saleh Ahmed, S.M., 2019. Determination of muon absorption coefficients in heavy metal elements. Journal of Radiation Research and Applied Sciences, 12(1), pp.281-288.

[26] T. Sato et al., "Features of Particle and Heavy Ion Transport code System (PHITS) version 3.02," J. Nucl. Sci. Technol., vol. 55, no. 6, pp. 684-690, Jun. 2018, doi: 10.1080/00223131.2017.1419890. 